\title{
TREATMENT RESPONSE PREDICTION IN HEPATITIS C PATIENTS USING MACHINE LEARNING TECHNIQUES
}

\author{
Ashfaq Ali Kashif ${ }^{1}$, Birra Bakhtawar ${ }^{1}$, Asma Akhtar ${ }^{1}$, Samia Akhtar ${ }^{1}$, Nauman Aziz ${ }^{2}$, \\ Muhammad Sheraz Javeid ${ }^{3}$ \\ ${ }^{1}$ Department of Computer Science, Virtual University of Pakistan, Lahore, Pakistan \\ (ashfaq.ali00@gmail.com, birra.bakhtawar@gmail.com, asmaakhtarjanjua@gmail.com, \\ samiaakhtar9898@gmail.com) \\ ${ }^{2}$ Department of Computer Science, Superior University, Lahore, Pakistan, na.pitafi@ gmail.com \\ ${ }^{3}$ School of Computer Science, National College of Business Administration \& Economics, Lahore, \\ Pakistan, sherazjaveid@gmail.com
}

\begin{abstract}
:
The proper prognosis of treatment response is crucial in any medical therapy to reduce the effects of the disease and of the medication as well. The mortality rate due to hepatitis c virus (HCV) is high in Pakistan as well as all over the world. During the treatment of any disease, prediction of treatment response against any particular medicine is difficult. This paper focuses on predicting the treatment response of a drug: "L-ornithine L-Aspartate (LOLA)" in hepatitis c patients. We have used various machine learning techniques for the prediction of treatment response, including: "K Nearest Neighbor, kStar, Naive Bayes, Random Forest, Radial Basis Function, PART, Decision Tree, OneR, Support Vector Machine and Multi-Layer Perceptron”. Performance measures used to analyze the performance of used machine learning techniques include, “Accuracy, Recall, Precision, and F-Measure".
\end{abstract}

Keywords-Treatment Response Prediction, Hepatis C, Machine Learning, Medical Data Mining 


\section{INTRODUCTION}

Hepatitis is a dangerous and transmissible disease [1-4]. The virus of this disease can spread from one infected person to another healthy human being. This disease has already infected almost 17 million people in all over the world and the numbers are getting increased day by day [2-6]. The virus of hepatitis c needs to be treated as early as possible to control and reduce the effects of the disease. A proper and complete medical therapy is needed in order to bring down the effects of this disease. However, not one medical therapy is good for all the patients. Same medicine may have different effects on different people due to other known or hidden medical reasons of the patients [5-10]. This paper explores the importance of machine learning techniques to predict the treatment response of a drug: "L-ornithine L-Aspartate (LOLA)" in hepatitis c patients. Various machine learning techniques are used in this study for the prediction of treatment response, including: "K Nearest Neighbor, kStar, Naive Bayes, Random Forest, Radial Basis Function, PART, Decision Tree, OneR, Support Vector Machine and Multi-Layer Perceptron”. Performance of used machine learning techniques is analyzed and evaluated by various measures, including: “Accuracy, Recall, Precision, and F-Measure".

\section{RELATED WORK}

Many researchers have used machine learning and data mining techniques in order to predict the treatment response. Researchers in [11] has built a hybrid framework to examine the similarity of drugs response using advanced K-means clustering. Researchers in [12] predict the response of Clozapine; a drug used for the treatment of psychiatric disease. They used a machine learning approach to predict the response of drug. In [13], a machine learning supported framework built by the team of researchers on post-marketing dataset for predicting the Anti-PD-1 treatment response. In [2], treatment response prediction is performed using the Artificial Neural Network and Decision Tree. In [14], researchers used Decision Tree (DT) to predict the early diagnosis of hepatitis $\mathrm{C}$ in the diabetic patients using the routine laboratory tests. In [15], researchers used different machine learning techniques to predict the drugs toxicity and its side effects, these side effects weaken the quality of life, which needs to be addressed on priority bases. In [16], researchers presented a machine learning based prediction for HIV medication resistance with a set of mutant features. The proposed algorithm first identify the protein structure then classify it 
based on sparse representation using Artificial Neural Network, Support Vector Machine and Regression. In [17], the researchers explored that the deep learning techniques played a vital role in cancer patients for identification the drugs response. The researchers critically examined the cancer cell in order to predict the drug response on them. Researchers in [18] uses Bayesian Network for predicting the esophageal disease which is an adverse effect, present in the disease of liver cirrhosis.

\section{MATERIALS AND METHODS}

This study explores the effectiveness of machine learning techniques in the prediction of treatment response in hepatitis c patients. Machine learning and data mining techniques have been widely and effectively used by many researchers in various domains and fields including: Sentiment/Polarity analysis [19-25], Rainfall/Weather Prediction [26-27], and Network Intrusion Detection/Network Security [28-29], Software Defect Prediction [30-38], Medical and Health data mining [39-47]. Machine learning techniques included in this study for the prediction of treatment response are: “K Nearest Neighbor, kStar, Naive Bayes, Random Forest, Radial Basis Function, PART, Decision Tree, OneR, Support Vector Machine and Multi-Layer Perceptron”. The machine learning techniques are used on the patient's dataset collected from a hospital in city of Lahore, Pakistan. The dataset consists of various attributes regarding the medical information of the patient. The attribute which is predicted on the basis of medical information is the response, which consists of two categorical values: Respondent or Not Respondent. This attribute reflects that the particular patient is showing response against LOLA therapy or not. The used dataset is preprocessed before the classification. The pre-processing activities include: cleaning and normalization (Fig 1). 


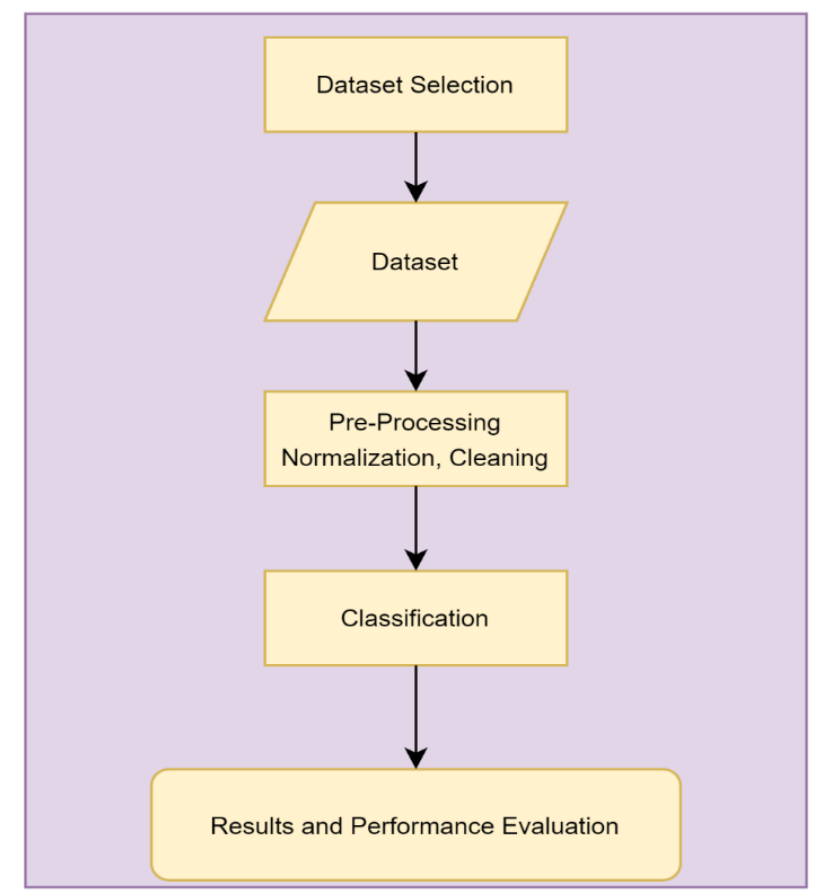

Fig 2 Treatment Response Prediction using Machine Learning Techniques

\section{RESULTS AND DISCUSSIONS}

Evaluating the performance of used machine learning techniques is a crucial stage where we have to compare the accuracy measures of used algorithms in order to select the best one for future use [48-50]. Performance of used machine learning techniques is analyzed and evaluated by various measures, such as: "Accuracy, Recall, Precision, and F-Measure". The parameters used in the formulas of performance measures came from the confusion matrix (Fig 2), which is the ultimate result of classification/prediction. The Parameters used in the confusion matrix are: TP, FN, TN and FP [30-38].

The formulas of the TP, FN, TN and FP has given below.

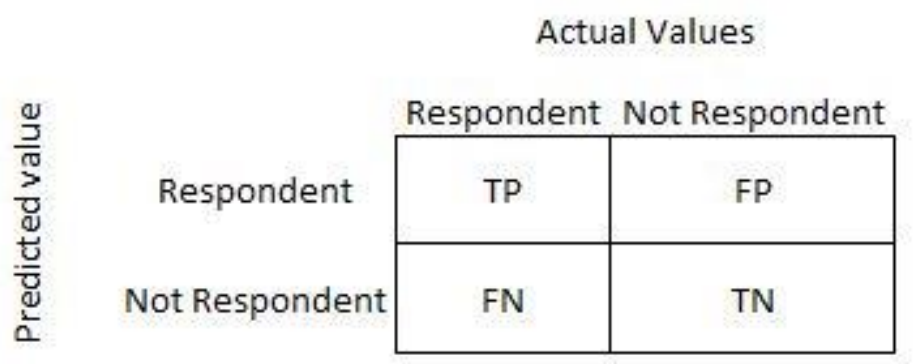

Fig 2 Confusion Matrix 


$$
\begin{aligned}
& \text { Precision }=\frac{T P}{(T P+F P)} \\
& \text { Recall }=\frac{T P}{(T P+F N)} \\
& F-\text { Measures }=\frac{\text { Precisin } * \text { Recall } * 2}{(\text { Precisin }+ \text { Recall })} \\
& \text { Accuracy }=\frac{T P+T N}{T P+T N+F P+F N}
\end{aligned}
$$

The Weka tool is used to conduct the experiments. All of the used performance measures are provided by the WEKA tool. Table 1 reflects the results on training dataset with all of the used classification algorithms.

Table 1: Results with Training Dataset

\begin{tabular}{|c|l|c|c|c|}
\hline Classifier & \multicolumn{1}{|c|}{ Class } & Precision & Recall & F-Measure \\
\hline \multirow{2}{*}{ NB } & Respondent & 0.893 & 0.895 & 0.894 \\
\cline { 2 - 5 } & Not Respondent & 0.622 & 0.618 & 0.62 \\
\hline \multirow{3}{*}{ MLP } & Respondent & 0.914 & 0.953 & 0.933 \\
\cline { 2 - 5 } & Not Respondent & 0.803 & 0.681 & 0.737 \\
\hline \multirow{2}{*}{ RBF } & Respondent & 0.878 & 0.922 & 0.9 \\
\cline { 2 - 5 } & Not Respondent & 0.661 & 0.542 & 0.595 \\
\hline \multirow{2}{*}{ SVM } & Respondent & 0.89 & 0.922 & 0.906 \\
\cline { 2 - 5 } & Not Respondent & 0.68 & 0.59 & 0.632 \\
\hline \multirow{2}{*}{ KNN } & Respondent & 0.963 & 0.901 & 0.931 \\
\cline { 2 - 5 } & Not Respondent & 0.712 & 0.875 & 0.785 \\
\hline \multirow{2}{*}{ K* } & Respondent & 0.924 & 0.944 & 0.934 \\
\cline { 2 - 5 } & Not Respondent & 0.782 & 0.722 & 0.751 \\
\hline \multirow{2}{*}{ OneR } & Respondent & 0.892 & 0.911 & 0.901 \\
\cline { 2 - 5 } & Not Respondent & 0.654 & 0.604 & 0.628 \\
\hline \multirow{2}{*}{ PART } & Respondent & 0.931 & 0.913 & 0.922 \\
\cline { 2 - 5 } & Not Respondent & 0.708 & 0.757 & 0.732 \\
\hline \multirow{2}{*}{ DT } & Respondent & 0.692 & 0.625 & 0.657 \\
\cline { 2 - 5 } & Not Respondent & 0.898 & 0.922 & 0.91 \\
\hline \multirow{2}{*}{ RF } & Respondent & 0.931 & 0.936 & 0.933 \\
\cline { 2 - 5 } & Not Respondent & 0.766 & 0.75 & 0.758 \\
\hline
\end{tabular}

Table 2 shows the results on testing dataset. It can be seen that the accuracy measures are different 
on both the datasets.

Table 2: Results with Testing Dataset

\begin{tabular}{|c|l|c|c|c|}
\hline Classifier & \multicolumn{1}{|c|}{ Class } & Precision & Recall & F-Measure \\
\hline \multirow{2}{*}{ NB } & Respondent & 0.885 & 0.865 & 0.875 \\
\cline { 2 - 5 } & Not Respondent & 0.552 & 0.597 & 0.574 \\
\hline \multirow{3}{*}{ MLP } & Respondent & 0.873 & 0.865 & 0.869 \\
\cline { 2 - 5 } & Not Respondent & 0.531 & 0.548 & 0.54 \\
\hline \multirow{2}{*}{ RBF } & Respondent & 0.882 & 0.91 & 0.896 \\
\cline { 2 - 5 } & Not Respondent & 0.636 & 0.565 & 0.598 \\
\hline \multirow{2}{*}{ SVM } & Respondent & 0.873 & 0.932 & 0.902 \\
\cline { 2 - 5 } & Not Respondent & 0.681 & 0.516 & 0.587 \\
\hline \multirow{2}{*}{ KNN } & Respondent & 0.9 & 0.811 & 0.853 \\
\cline { 2 - 5 } & Not Respondent & 0.5 & 0.677 & 0.575 \\
\hline \multirow{2}{*}{ K* } & Respondent & 0.898 & 0.874 & 0.886 \\
\cline { 2 - 5 } & Not Respondent & 0.588 & 0.645 & 0.615 \\
\hline \multirow{2}{*}{ OneR } & Respondent & 0.888 & 0.932 & 0.91 \\
\cline { 2 - 5 } & Not Respondent & 0.706 & 0.581 & 0.637 \\
\hline \multirow{2}{*}{ PART } & Respondent & 0.9 & 0.851 & 0.875 \\
\cline { 2 - 5 } & Not Respondent & 0.554 & 0.661 & 0.603 \\
\hline \multirow{2}{*}{ DT } & Respondent & 0.889 & 0.937 & 0.912 \\
\cline { 2 - 5 } & Not Respondent & 0.72 & 0.581 & 0.643 \\
\hline \multirow{2}{*}{ RF } & Respondent & 0.895 & 0.883 & 0.889 \\
\cline { 2 - 5 } & Not Respondent & 0.6 & 0.629 & 0.614 \\
\hline & & & & \\
\hline
\end{tabular}

Accuracy of the training dataset and testing dataset is reflected in Table 3. The accuracy in the training dataset is highest in $\mathrm{KNN}, \mathrm{K}^{*}$ and Random Forest. On the other hand, Decision Tree shows the highest accuracy in the test dataset.

Table 3: Accuracy Comparison

\begin{tabular}{|c|c|c|}
\hline Classifier & Training Accuracy & Test Accuracy \\
\hline NB & 83.4598 & 80.6338 \\
\hline MLP & 89.3778 & 79.5775 \\
\hline RBF & 83.915 & 83.4507 \\
\hline SVM & 84.9772 & 84.1549 \\
\hline KNN & 89.5296 (Highest) & 78.169 \\
\hline K $^{*}$ & 89.5296 (Highest) & 82.3944 \\
\hline OneR & 84.3703 & 85.5634 \\
\hline PART & 87.8604 & 80.9859 \\
\hline DT & 85.736 & 85.9155 (Highest) \\
\hline RF & 89.5296 (Highest) & 82.7465 \\
\hline
\end{tabular}




\section{CONCLUSION:}

This paper presented a comparative analysis of various machine learning techniques on the prediction of treatment response in hepatitis c patients. The machine learning techniques used in this study include: "K Nearest Neighbor, kStar, Naive Bayes, Random Forest, Radial Basis Function, PART, Decision Tree, OneR, Support Vector Machine and Multi-Layer Perceptron”. The performance of these algorithms is measures by different evaluation measures such as " $\mathrm{F}$ measures, Precision, Accuracy and Recall". It is observed that in the accuracy measure, training data, KNN, $\mathrm{K}^{*}$ and RF performed well where as in test data DT performed well.

\section{REFERENCES}

[1] Yasin, H., Jilani, T. A., \& Danish, M. (2011). Hepatitis-C classification using data mining techniques. International Journal of Computer Applications, 24(3), 1-6.

[2] ElHefnawi, M., Abdalla, M., Ahmed, S., Elakel, W., Esmat, G., Elraziky, M., ... \& Hassan, M. (2012, August). Accurate prediction of response to Interferon-based therapy in Egyptian patients with Chronic Hepatitis C using machine-learning approaches. In 2012 IEEE/ACM International Conference on Advances in Social Networks Analysis and Mining (pp. 771-778). IEEE.

[3] Pawlotsky, J. M., Negro, F., Aghemo, A., Berenguer, M., Dalgard, O., Dusheiko, G., ... \& European Association for the Study of the Liver. (2020). EASL recommendations on treatment of hepatitis C: final update of the series is. Journal of Hepatology, 73(5), 1170-1218.

[4] Butterworth, R. F., \& McPhail, M. J. (2019). L-Ornithine L-Aspartate (LOLA) for hepatic encephalopathy in cirrhosis: results of randomized controlled trials and meta-analyses. Drugs, 79(1), 31-37.

[5] World Health Organization. (2021). Recommendations and guidance on hepatitis C virus selftesting. World Health Organization. https://apps.who.int/iris/handle/10665/342803. License: CC BY-NC-SA 3.0 IGO

[6] World Health Organization, "Hepatitis C [Hepatitis C], Hepatitis C (who.int)

[7] Stoean, R., Stoean, C., Lupsor, M., Stefanescu, H., \& Badea, R. (2011). Evolutionary-driven support vector machines for determining the degree of liver fibrosis in chronic hepatitis C. Artificial intelligence in medicine, 51(1), 53-65.

[8] Huang, R., Rao, H., Yang, M., Gao, Y., Wang, J., Jin, Q., ... \& Wei, L. (2020). Noninvasive measurements predict liver fibrosis well in hepatitis $\mathrm{C}$ virus patients after direct-acting antiviral therapy. Digestive diseases and sciences, 65(5), 1491-1500.

[9] Mohamed, A. A., Elbedewy, T. A., El-Serafy, M., El-Toukhy, N., Ahmed, W., \& El Din, Z. A. (2015). Hepatitis C virus: A global view. World journal of hepatology, 7(26), 2676. 
[10] Shen, J., Zhang, C. J., Jiang, B., Chen, J., Song, J., Liu, Z., ... \& Ming, W. K. (2019). Artificial intelligence versus clinicians in disease diagnosis: systematic review. JMIR medical informatics, 7(3), e10010.

[11] Menon, M. S. (2021). A Hybrid Framework For Drug Response Similarity Opting Machine Learning Approach. Turkish Journal of Computer and Mathematics Education (TURCOMAT), 12(11), 550-558.

[12] Ciprian, C., Masychev, K., Ravan, M., Reilly, J. P., \& Maccrimmon, D. (2020). A Machine Learning Approach Using Effective Connectivity to Predict Response to Clozapine Treatment. IEEE Transactions on Neural Systems and Rehabilitation Engineering, 28(12), 2598-2607.

[13] Liu, X., Xiao, Z., Song, Y., Zhang, R., Li, X., \& Du, Z. (2021). A Machine Learning-Aided Framework to Predict Outcomes of Anti-PD-1 Therapy for Patients With Gynecological Cancer on Incomplete Post-Marketing Surveillance Dataset. IEEE Access, 9, 120464-120480.

[14] Saad, Y., Awad, A., Alakel, W., Doss, W., Awad, T., \& Mabrouk, M. (2018). Data mining of routine laboratory tests can predict liver disease progression in Egyptian diabetic patients with hepatitis C virus (G4) infection: a cohort study of 71806 patients. European journal of gastroenterology \& hepatology, 30(2), 201-206.

[15] Khan, M. (2017). Drug side-effect prediction using machine learning methods.

[16] Yu, X. (2014). HIV drug resistant prediction and featured mutants selection using machine learning approaches.

[17] Baptista, D., Ferreira, P. G., \& Rocha, M. (2021). Deep learning for drug response prediction in cancer. Briefings in bioinformatics, 22(1), 360-379.

[18] Abd El-Salam, S. M., Ezz, M. M., Hashem, S., Elakel, W., Salama, R., ElMakhzangy, H., \& ElHefnawi, M. (2019). Performance of machine learning approaches on prediction of esophageal varices for Egyptian chronic hepatitis C patients. Informatics in Medicine Unlocked, 17, 100267.

[19] Ahmad, M., Aftab, S., Muhammad, S. S., \& Waheed, U. (2017). Tools and techniques for lexicon driven sentiment analysis: a review. Int. J. Multidiscip. Sci. Eng, 8(1), 17-23.

[20] Ahmad, M., Aftab, S., Muhammad, S. S., \& Ahmad, S. (2017). Machine learning techniques for sentiment analysis: A review. Int. J. Multidiscip. Sci. Eng, 8(3), 27.

[21] Ahmad, M., Aftab, S., Ali, I., \& Hameed, N. (2017). Hybrid tools and techniques for sentiment analysis: a review. Int. J. Multidiscip. Sci. Eng, 8(3), 29-33.

[22] Ahmad, M., \& Aftab, S. (2017). Analyzing the Performance of SVM for Polarity Detection with Different Datasets. International Journal of Modern Education \& Computer Science, 9(10).

[23] Ahmad, M., Aftab, S., \& Ali, I. (2017). Sentiment analysis of tweets using svm. Int. J. Comput. Appl, 177(5), 25-29.

[24] Ahmad, M., Aftab, S., Bashir, M. S., \& Hameed, N. (2018). Sentiment analysis using SVM: A systematic literature review. International Journal of Advanced Computer Science and Applications, 9(2), 182-188.

[25] Ahmad, M., Aftab, S., Bashir, M. S., Hameed, N., Ali, I., \& Nawaz, Z. (2018). SVM optimization for sentiment analysis. Int. J. Adv. Comput. Sci. Appl, 9(4), 393-398.

[26] Shabib Aftab, M. A., Hameed, N., Bashir, M. S., Ali, I., \& Nawaz, Z. (2018). Rainfall prediction in lahore city using data mining techniques. International journal of advanced computer science and applications, 9(4).

[27] Shabib Aftab, M. A., Hameed, N., Bashir, M. S., Ali, I., \& Nawaz, Z. Rainfall Prediction using Data Mining Techniques: A Systematic Literature Review. 
[28] Iqbal, A., \& Aftab, S. (2019). A Feed-Forward and Pattern Recognition ANN Model for Network Intrusion Detection. International Journal of Computer Network \& Information Security, 11(4).

[29] Iqbal, A., Aftab, S., Ullah, I., Saeed, M. A., \& Husen, A. (2019). A Classification Framework to Detect DoS Attacks. International Journal of Computer Network \& Information Security, 11(9).

[30] Iqbal, A., Aftab, S., Ali, U., Nawaz, Z., Sana, L., Ahmad, M., \& Husen, A. (2019). Performance analysis of machine learning techniques on software defect prediction using NASA datasets. Int. J. Adv. Comput. Sci. Appl, 10(5), 300-308.

[31] Iqbal, A., Aftab, S., Ullah, I., Bashir, M. S., \& Saeed, M. A. (2019). A feature selection based ensemble classification framework for software defect prediction. International Journal of Modern Education and Computer Science, 11(9), 54.

[32] Iqbal, A., Aftab, S., \& Matloob, F. (2019). Performance analysis of resampling techniques on class imbalance issue in software defect prediction. Int. J. Inf. Technol. Comput. Sci, 11(11), 44-53.

[33] Matloob, F., Aftab, S., \& Iqbal, A. (2019). A Framework for Software Defect Prediction Using Feature Selection and Ensemble Learning Techniques. International Journal of Modern Education \& Computer Science, 11(12).

[34] Iqbal, A., \& Aftab, S. (2020). A Classification Framework for Software Defect Prediction Using Multi-filter Feature Selection Technique and MLP. International Journal of Modern Education \& Computer Science, 12(1).

[35] Iqbal, A., \& Aftab, S. (2020). Prediction of Defect Prone Software Modules using MLP based Ensemble Techniques. International Journal of Information Technology and Computer Science, 12(3), 26-31.

[36] Ali, U., Aftab, S., Iqbal, A., Nawaz, Z., Bashir, M. S., \& Saeed, M. A. (2020). Software Defect Prediction Using Variant based Ensemble Learning and Feature Selection Techniques. International Journal of Modern Education \& Computer Science, 12(5).

[37] Matloob, F., Aftab, S., Ahmad, M., Khan, M. A., Fatima, A., Iqbal, M., ... \& Elmitwally, N. S. Software Defect Prediction Using Supervised Machine Learning Techniques: A Systematic Literature Review.

[38] Matloob, F., Ghazal, T. M., Taleb, N., Aftab, S., Ahmad, M., Khan, M. A., ... \& Soomro, T. R. (2021). Software Defect Prediction Using Ensemble Learning: A Systematic Literature Review. IEEE Access.

[39] Daoud, M. S., Aftab, S., Ahmad, M., Khan, M. A., Iqbal, A., Abbas, S., ... \& Ihnaini, B. (2022). Machine Learning Empowered Software Defect Prediction System.

[40] Aftab, S., Alanazi, S., Ahmad, M., Khan, M. A., Fatima, A., \& Elmitwally, N. S. (2021). CloudBased Diabetes Decision Support System Using Machine Learning Fusion.

[41] Ahmad, M., Alfayad, M., Aftab, S., Khan, M. A., Fatima, A., Shoaib, B., ... \& Elmitwally, N. S. (2021). Data and Machine Learning Fusion Architecture for Cardiovascular Disease Prediction. CMC-COMPUTERS MATERIALS \& CONTINUA, 69(2), 2717-2731.

[42] Siddiqui, S. Y., Haider, A., Ghazal, T. M., Khan, M. A., Naseer, I., Abbas, S., ... \& Ateeq, K. (2021). IoMT Cloud-based Intelligent Prediction of Breast Cancer Stages Empowered with Deep Learning. IEEE Access.

[43] Ghazal, T. M., Abbas, S., Munir, S., Khan, M. A., Ahmad, M., Issa, G. F., ... \& Hasan, M. K. (2022). Alzheimer Disease Detection Empowered with Transfer Learning. 
[44] Ihnaini, B., Khan, M. A., Khan, T. A., Abbas, S., Daoud, M. S., Ahmad, M., \& Khan, M. A. (2021). A Smart Healthcare Recommendation System for Multidisciplinary Diabetes Patients with Data Fusion Based on Deep Ensemble Learning. Computational Intelligence and Neuroscience, 2021.

[45] Siddiqui, S. Y., Abbas, S., Khan, M. A., Naseer, I., Masood, T., Khan, K. M., ... \& Almotiri, S. H. (2021). Intelligent decision support system for COVID-19 empowered with deep learning. CMCComput. Mater. Continua, 66(2), 1719-1732.

[46] Khan, T. A., Abbas, S., Ditta, A., Khan, M. A., Alquhayz, H., Fatima, A., \& Khan, M. F. (2020). IoMT-based smart monitoring hierarchical fuzzy inference system for diagnosis of covid-19. Computers, Materials and Continua, 65(3), 2591-2605.

[47] Khan, M. A., Abbas, S., Atta, A., Ditta, A., Alquhayz, H., Khan, M. F., \& Naqvi, R. A. (2020). Intelligent cloud based heart disease prediction system empowered with supervised machine learning.

[48] Ghazal, T. M., Noreen, S., Said, R. A., Khan, M. A., Siddiqui, S. Y., Abbas, S., ... \& Ahmad, M. (2022). Energy Demand Forecasting Using Fused Machine Learning Approaches.

[49] Daoud, M. S., Fatima, A., Khan, W. A., Khan, M. A., Abbas, S., Ihnaini, B., ... \& Aftab, S. (2022). Joint Channel and Multi-User Detection Empowered with Machine Learning.

[50] Aziz, N., \& Aftab, S. (2021). Data Mining Framework for Nutrition Ranking: Methodology: SPSS Modeller. International Journal of Technology, Innovation and Management (IJTIM), 1(1), 85-95.

[51] Ali, N., Ghazal, T., Ahmed, A., Abbas, S., Khan, M., Alzoubi, H., Farooq, U., Ahmed, M. \& Khan, M. (2021) Fusion-Based Supply Chain Collaboration Using Machine Learning Techniques. Intelligent Automation \& Soft Computing, 31(3), 1671-1687

[52] Ghazal, T., Hasan, M., Alshurideh M., Alzoubi, H., Ahmad, M., Akbar, S., Al Kurdi, B. \& Akour, I. (2021) IoT for Smart Cities: Machine Learning Approaches in Smart Healthcare-A Review, Future Internet, 13, 218. https://doi.org/10.3390/fi13080218

[53] Ali, N., Ahmed, A., Anum, L., Ghazal, T., Abbas, S., Khan, M., Alzoubi, H. \& Ahmad, A. (2021) Modelling Supply Chain Information Collaboration Empowered with Machine Learning Technique. Intelligent Automation \& Soft Computing, 30(1): 243-257. DOI:10.32604/iasc.2021.018983

[54] Analyzing the effect of knowledge management processes in the services' quality in Iraqi commercial banks. International Review of Management and Business Research, 5(1), 302-314. (2016)

[55] Exploring the Impact of the use of Business Information systems BIS on the organizational performance effectiveness. International Journal of Business and Management Invention, 5(4), 4855. (2016)

[56] The Impact of Managers Efficiency on Quality of Strategic Decision-making under Crisis Management. European Journal of Business and Management, 7(26), 156-166. (2015)

[57] Investigating the Relationship between Knowledge Management Processes and Organizational Performance: The Mediating Effect of Organizational Innovation. International Review of Management and Business Research, 4(4), 977-997 (2015).

[58] The Impact of Business Process Management on Business Performance Superiority. International Journal of Business and Management Review, 3(2), 17-34 (2015)

[59] Evaluating Strategic Quality Management Dimensions Using Analytic Hierarchy Process (AHP) and its Impact on Organizational Success. International Journal of Research in Management, 5(1), $137-150$ (2015) 
[60] American Muslims' Perceptions Toward Transforming Islamic Banking System. International Journal of Economics, Commerce and Management, 5(1), 1-16 (2015)

[61] Exploring the Relationship between Quality Orientation, New Services Development and Organizational Performance. American Academic \& Scholarly Research Journal, 5(3), 315-329 (2013)

[62] Analyze the Impact of Managers Awareness of Environmental Uncertainty on Exploiting Strategic Competencies. Egyptian Journal for Commercial Studies, 34(2), 611-625 (2010)

[63] Applying Electronic Supply Chain Management Using Multi-Agent System: A Managerial Perspective. International Arab Journal of e-Technology, 1(3), 106-113 (2010)

[64] Propose a model for Performance Criteria and measuring its impact for Achieving Excellence, Association of Arab Universities Journal, 56 (4), 920-941. (2010)

[65] S. Y. Siddiqui, A. Haider, T. M. Ghazal, M. A. Khan, I. Naseer, S. Abbas, M. Rahman, J. A. Khan, M. Ahmad, M. K. Hasan, A. M. A, and K. Ateeq, "IOMT cloud-based intelligent prediction of breast cancer stages empowered with Deep Learning," IEEE Access, vol. 9, pp. 146478-146491, Oct. 2021.

[66] S. Abbas, Y. Alhwaiti, A. Fatima, M. A. Khan, M. Adnan Khan, T. M. Ghazal, A. Kanwal, M. Ahmad, and N. Sabri Elmitwally, "Convolutional neural network based intelligent handwritten document recognition," Computers, Materials \& Continua, vol. 70, no. 3, pp. 4563-4581, Oct. 2021.

[67] T. M. Ghazal, S. Abbas, S. Munir, M. A. Khan, M. Ahmad, G. F. Issa, S. Binish Zahra, M. Adnan Khan, and M. Kamrul Hasan, "Alzheimer disease detection empowered with transfer learning," Computers, Materials \& Continua, vol. 70, no. 3, pp. 5005-5019, Oct. 2021.

[68] N. Ali, T. M. Ghazal, A. Ahmed, S. Abbas, M. A. Khan, H. M. Alzoubi, U. Farooq, M. Ahmad, and M. Adnan Khan, "Fusion-based supply chain collaboration using Machine Learning Techniques," Intelligent Automation \& Soft Computing, vol. 31, no. 3, pp. 1671-1687, Oct. 2021.

[69] M. K. Hasan, T. M. Ghazal, A. Alkhalifah, K. A. Abu Bakar, A. Omidvar, N. S. Nafi, and J. I. Agbinya, "Fischer linear discrimination and quadratic discrimination analysis-based data mining technique for internet of things framework for Healthcare," Frontiers in Public Health, vol. 9, Oct. 2021.

[70] R. Bibi, Y. Saeed, A. Zeb, T. M. Ghazal, T. Rahman, R. A. Said, S. Abbas, M. Ahmad, and M. A. Khan, "Edge AI-based automated detection and classification of road anomalies in VANET using Deep Learning," Computational Intelligence and Neuroscience, vol. 2021, pp. 1-19, Sep. 2021.

[71] M. Adnan Khan, T. M. Ghazal, S.-W. Lee, and A. Rehman, "Data Fusion-based machine learning architecture for intrusion detection," Computers, Materials \& Continua, vol. 70, no. 2, pp. 33993413, Sep. 2021. 